\title{
Adverse childhood experiences and multiple mental health outcomes through adulthood: A prospective birth cohort study ${ }^{\text {ir }}$
}

\author{
Dawid Gondek $^{\mathrm{a}, *}$, Praveetha Patalay ${ }^{\mathrm{b}, \mathrm{c}}$, Rebecca E. Lacey ${ }^{\mathrm{a}}$ \\ ${ }^{a}$ Research Department of Epidemiology and Public Health, University College London, London, England, UK \\ ${ }^{\mathrm{b}}$ Centre for Longitudinal Studies, Department of Social Science, UCL Institute of Education, University College London, London, England, UK \\ ${ }^{\mathrm{c}}$ MRC Unit for Lifelong Health and Ageing, Department of Population Science and Experimental Medicine, University College London, London, England, UK
}

\section{A R T I C L E I N F O}

\section{Keywords:}

Adverse childhood experiences

Mental health

Psychological distress

National child development study

\begin{abstract}
A B S T R A C T
Objective: Exposure to adverse childhood experiences (ACEs) is associated with a broad range of mental healthrelated outcomes. Previous studies tended to use retrospectively reported ACEs, measure mental health outcomes at a one time point in adulthood and focus on individual outcomes. Hence, this study aimed to examine the association between prospectively and retrospectively measured ACEs and a wide range of mental health-related outcomes, spanning ages 16 to55, using a prospective birth cohort, representative of those born around 1958 in Great Britain.

Methods: The study used the 1958 National Child Development Study ( $\mathrm{n}=7980)$. Adverse childhood experiences were measured both prospectively and retrospectively, and combined into ACE scores. The associations between the ACE scores and mental health were quantified with linear regression for continuous and robust Poisson regression for binary outcomes.

Results: We found a dose-response association between prospectively and retrospectively reported ACEs and all studied mental health-related outcomes, after accounting for multiple covariates. Among those with $2+$ (vs 0 ) prospective ACEs, the risk of clinically significant psychological distress was up to 2.14 times higher, and of seeing a mental health specialist up to 2.85 times higher.

Conclusions: Our findings reiterate the need for early-life interventions to reduce inequalities in mental health.
\end{abstract}

\section{Introduction}

Exposure to adverse childhood experiences (ACEs) is associated with a broad range of mental health-related outcomes (Ege et al., 2015; Hughes et al., 2016, 2017; Selous et al., 2020). Cumulatively, adverse childhood experiences can lead to dysregulating neurological and physiological systems, for instance, associated with chronic stress, which in turn results in increased vulnerability to poor mental health and wellbeing (McLaughlin et al., 2019). Early adversities can also initiate a chain of cumulative risks that eventually lead to poor mental health (Kuh \& the New Dynamics of Ageing Preparatory Network, 2007). For instance, children exposed to neglect, which is associated with a lack of expected cognitive and social inputs, may suffer from poorer cognitive abilities that can translate into limited educational and occupational opportunities (England, 2019; McLaughlin et al., 2019; Miller et al., 2018; Peng
$\&$ Kievit, 2020). These, in turn, are often associated with worse wellbeing and mental health (Allen et al., 2014).

The main limitation of previous studies examining the link between ACEs and mental health is that they tended to rely on retrospective reports of ACEs, which potentially suffer from recall bias. Moreover, studies typically included outcomes measured at one time point in adulthood, precluding understanding of the persistence of any impacts of ACEs on mental health across adulthood (Hughes et al., 2017). Besides, existing longitudinal research on ACEs is focused mainly on mental ill-health in the form of general psychological distress and it tends to be limited to studying individual mental health outcomes (Hughes et al., 2017; Kalmakis \& Chandler, 2015; Selous et al., 2020). Hence, the impact of ACEs on other aspects of mental health throughout adulthood is less known, for instance, wellbeing, utilisation of mental health services, or taking psychotropic medications (Kalmakis \& Chandler, 2015; Selous et al., 2020).

\footnotetext{
The manuscript has been published as a preprint (MEDRXIV/2021/252273).

* Corresponding author. Institute of Epidemiology \& Health Care, Research Department of Epidemiology \& Public Health, University College London, 1-19 Torrington Pl, Fitzrovia, London, WC1E 7HB, UK.

E-mail address: dawid.gondek.14@ucl.ac.uk (D. Gondek).
} 
These limitations can be addressed by taking advantage of rich longitudinal data available from the 1958 National Child Development Study (NCDS) (Power \& Elliott, 2006), a prospective birth cohort, representative of those born around 1958 in Great Britain. The outcomes available in the NCDS include utilisation of mental health services, taking psychotropic medications, standardised measures of psychological distress, life satisfaction, quality of life, and self-reported general health (as it tends to correlate strongly with mental health) (Chang-Quan et al., 2010). The use of healthcare and medications are vital outcomes from the public health perspective, as they capture the direct burden on health services. However, as health is not merely an absence of disease (Slade, 2010), it is important to understand whether ACEs also affect one's wellbeing. Following the approach of outcome-wide epidemiology (VanderWeele et al., 2020), including multiple outcomes representing the entire spectrum of mental health allows for greater efficiency for the reader, more rapid expansion of knowledge and a direct comparison of the relative magnitude of the association between ACEs and various domains of mental health, which to our knowledge has not been studied previously. The main objective of our study was to produce the most comprehensive analysis of the association between ACEs and mental health-related outcomes up to date - with ACEs reported both prospectively and retrospectively, outcomes including various indicators of both wellbeing and mental illness, which spanned four decades, while accounting for a wide range of confounding factors.

\section{Methods}

\subsection{Participants}

The NCDS follows the lives of 17,415 people born in England, Scotland, and Wales in a single week of 1958, and it is representative of the British population born around this year (Power \& Elliott, 2006). It includes information on physical and educational development, economic circumstances, employment, family life, health behaviours, wellbeing, social participation, and attitudes. The history, design, and features of the NCDS have been described elsewhere (Power \& Elliott, 2006). The NCDS has been granted ethical approval for each sweep from 2000 by the National Health Service (NHS) Research Ethics Committee. All participants provided written informed consent after a thorough explanation of the research procedures. We limited the sample of the NCDS to participants without missing information on retrospectively reported ACEs and based in Great Britain at the last data wave (at age 55) $(n=7980)$ (see eFig. 1 for sample flow diagram).

\subsection{Adverse childhood experiences}

ACEs were defined, based on previous studies using the NCDS, as traumatic and stressful psychosocial conditions that tend to co-occur, persist over time and are outside of the child's control (Kelly-Irving et al., 2013; Lacey et al., 2020). ACEs were measured prospectively through reports by parents (usually the mother; at ages 7-16), health visitors or teachers (at ages 7-16) and retrospectively by cohort members (at ages 33 and 44/45). Prospectively reported ACEs included parental separation/divorce, parental substance misuse, family conflict, death of a parent, parental mental health problems, physical neglect, and parental offending. Considering also retrospectively reported ACEs had the advantage of including different forms of abuse that were not measured prospectively in the cohort. In addition, prospective and retrospective measures of ACEs tend to identify different groups of individuals, with potentially varying pathways to adult mental health, hence it is important to test sensitivity of findings to both modes of reporting (Baldwin, Reuben, Newbury, \& Danese, 2019). The full list of retrospectively reported ACEs comprised parental separation/divorce, parental substance misuse, family conflict, witnessing abuse, parental mental health problems, sexual abuse, physical abuse, psychological abuse, and emotional neglect. See eTable 1 for more details on how they were measured.
We considered ACEs cumulatively as ACE scores. The prospective ACE score ranged from ' 0 ACEs' to '2 2 ACEs', and retrospective from ' 0 ACEs' to '4+ ACEs'. The analyses were also rerun using ACE scores as continuous variables.

\subsection{Mental health outcomes}

Continuous measures of psychological distress included the Malaise Inventory (at ages 23, 33, 42, 50), General Health Questionnaire (GHQ12; at age 42), depression and anxiety subscales of Clinical Interview Schedule - Revised (CIS-R; at age 44/45), psychological distress and wellbeing subscales of the Short Form Health Survey (SF-36; at age 50). Continuous measures of wellbeing comprised the Warwick-Edinburgh Mental Wellbeing Scale (WEMWBS; at age 50), and Quality of Life Scale (CASP; 12- item at age 50; 6-item at age 55) (Arseneault et al., 2020). All measures were standardised into $Z$ scores and wellbeing-oriented scales were reversed in line with higher scores on measures of psychological distress indicating worse mental health.

Binary outcomes included seeing a mental health specialist (at ages $16,23,33,42,5055$ ), poor/fair (vs good/very good/excellent) selfreported general health (at ages 23, 33, 42, 46, 50 55), being not at all/not very happy (vs fairly/very happy) (at age 33), indicators of clinically significant psychological distress - the Malaise Inventory (score $\geq 4$ ), GHQ (score $\geq 4$ ), CIS-R depression/anxiety (score $\geq 2$ ), SF-36 $($ score $\leq 52$ ), and WEMWBS (score $\leq 40$ ) (Arseneault et al., 2020). See eTable 2 for details on all outcome measures.

\subsection{Analytical strategy}

The associations between the ACE scores and mental health outcomes were studied with linear regression for continuous and robust Poisson regression for binary outcomes. The models were controlled for a range of child and family characteristics (see Fig. 1 for the list of covariates and eTable 3 for details of their measurement), which were likely to be associated with the exposure and outcome and were not on the causal pathway between these variables (VanderWeele, 2019).

The analyses used data after multiple imputation (MI) by chained equations, generating 20 datasets, which helps to reduce selection bias and maximise power (White et al., 2011). Detailed information on attrition and its determinants in the NCDS has been published elsewhere (Mostafa et al., 2021; Mostafa \& Wiggins, 2015). The characteristics of the samples with imputed data and complete cases only were very similar, however with a marginally higher proportion of those with $2+$ ACEs in the imputed sample (6.2\% vs $4.4 \%$ ) (see eTable 3 for more information). Participants with missing information on any outcome tended to be men, more socioeconomically disadvantaged (e.g., based on social class or housing tenure), and have more externalising problems at age 7 (see eTable 4 for determinants of having missing information on any outcome). More details on the missing data strategy applied in our analyses can be found in eAppendix 1 .

For all analyses, we combined men and women, as we found no evidence for differential ACEs-mental health associations between genders. The analysis was conducted in Stata 16 (StataCorp, 2020).

\section{Results}

Descriptive information about the study sample can be found in eTable 3.

We found strong evidence for a dose-response association between prospectively and retrospectively reported ACEs and all mental healthrelated outcomes, after accounting for multiple covariates (Fig. 1). All associations were "significant" at the conventional p-value threshold of 0.05 , whilst under Bonferroni correction, 35 tests out of 39 for prospective ACE score and 38 out of 39 for retrospective ACE score remained "significant" at an alternatve threshold of $\mathrm{p} \leq 0.001$. The magnitude of associations was relatively consistent regardless of ACEs being reported 
PANEL A
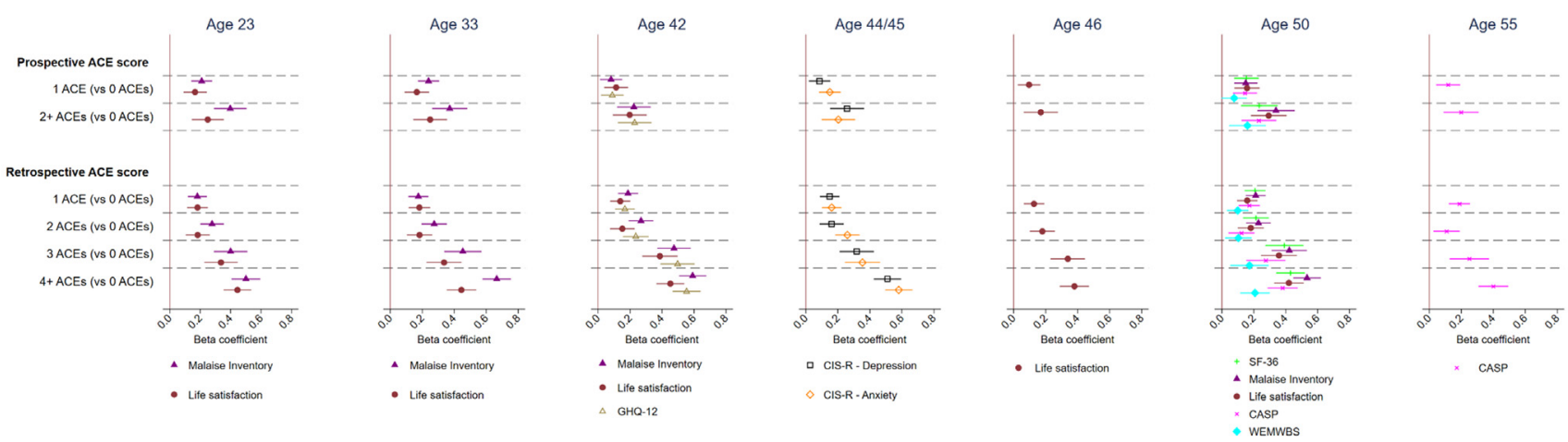

\section{PANEL B}
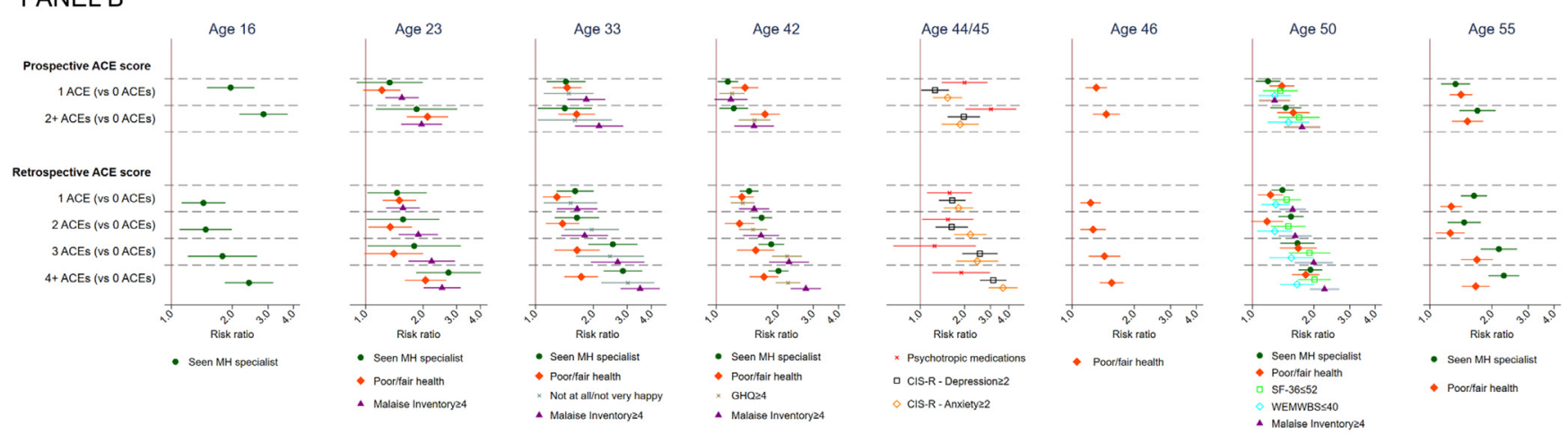

Fig. 1. The association between the ACE score and mental health - continuous (Panel A), binary (Panel B) outcomes.

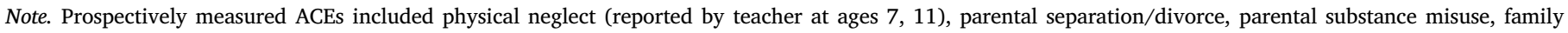
conflict, death of parent, parental mental health problems, parental offending (all reported by health visitor and/or parent at age 7-16).

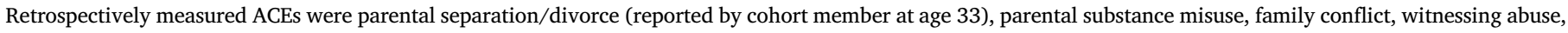
parental mental health problems, sexual abuse, physical abuse, psychological abuse, emotional neglect (all reported by cohort member at age 44/45).

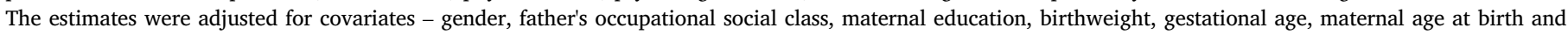
breastfeeding duration.

prospectively or retrospectively. For brevity, mainly findings on the prospective ACE score are discussed (see Fig. 1 for all results).

The prospective ACE score of $2+$ (vs 0 ) was associated with between 0.160 (95\% CI 0.044 to 0.276 ) and 0.293 (95\% CI 0.182 to 0.405 ) standard deviation (SD) higher score on measures of wellbeing, including life satisfaction, CASP, and WEMWBS. Scores on the measures of psychological distress, including the Malaise Inventory, CIS-R, GHQ-12, and SF-36, were between 0.203 (95 \% CI 0.099 to 0.308 ) and 0.398 (95\% CI 0.290 to 0.505$)$ SD higher. ACE score tended to have a somewhat stronger association with psychological distress than wellbeing when measured at the same age. For instance, at age 50 a retrospective ACE score of $4+$ (vs 0) was associated with 0.534 (95\% CI 0.448 to 0.621 ) SD higher score on the Malaise Inventory, compared with 0.208 (95\% CI 0.114 to 0.301 ) on WEMWBS. The correlations between measures within the same domains of mental health were moderate $(r=0.56-0.68$ for psychological distress and $r=0.49-0.69$ for wellbeing) when collected at the same age (see eTable 5 for all correlations). The correlations between wellbeing and distress measures tended to be weak $(r=0.16-0.54)$ when collected at the same age, with SF-36 having a moderate correlation with both wellbeing and distress measures $(r=0.52-71)$.

wThe prospective ACE score of $2+$ (vs 0 ) was associated with between $50 \%$ (risk ratio, 1.50, CI $95 \% 1.19$ to 1.90) and 2.14 times (CI $95 \% 1.60$ to 2.86 ) higher risk of having clinically significant psychological distress and up to 2.85 times (CI $95 \% 2.17$ to 3.75) higher risk of seeing a mental health specialist. The correlations between measures of distress and seeing a mental health specialist were moderate (rho $=0.45-0.63$ ) when collected at the same age. The correlations between measures indicating clinically significant distress were moderate to strong (rho $=0.62-0.80$ ).

Those with a prospective ACE score of $2+$ (vs 0 ) had also a 3 times higher risk of taking psychotropic medications at age 44/45 (CI $95 \%$ 2.03 to 4.50 ). Taking medications had a weak correlation with CIS-R depression $(r=0.17)$ or anxiety $(r=0.16)$. The prospective ACE score of $2+$ (vs 0 ) was associated with a $60 \%$ (CI $95 \% 1.03$ to 2.50 ) higher risk of not being happy at all or not very happy (vs fairly/very happy) at age 33 and with up to 2.11 times ( $95 \%$ CI 1.64 to 2.71 at age 23) higher risk of poor or fair (vs good/very good/excellent) self-rated general health. Having poor or fair self-rated general health had a weak to moderate association with clinically significant psychological distress (rho $=0.35-0.53$ ) and seeing a mental health specialist (rho $=0.32-0.56$ ).

The analyses were also rerun using ACE scores as continuous variables, producing highly comparable findings (see eTables 6 \& 7 for the estimates).

\section{Discussion}

We found that being exposed to ACEs is associated, in a dose-response fashion, with a range of mental health-related outcomes between age 16 and 55, thus replicating and extending previous research (Ege et al., 2015; Hughes et al., 2017; Selous et al., 2020). These associations appear to be somewhat stronger for psychological distress than wellbeing, are robust to rich covariates-adjustment, and are not sensitive to ACEs being measured prospectively or retrospectively, or outcomes being collected 
at specific ages. The effect sizes in our study for those with $4+$ retrospective ACEs were comparable to the ones obtained by the meta-analysis by Hughes and colleagues, for self-rated health, anxiety, depression, and life satisfaction (Hughes et al., 2017).

ACEs were found to have a strong relationship with a greater utilisation of healthcare services. This may be directly related to an increased need for these services due to elevated mental health problems. Individuals with experiences of ACEs might also have more contact with other social and health services, which could increase their likelihood of being referred for mental health support. However, there might also be other mechanisms at play that affect help-seeking behaviours, which overall would be expected to result in underestimating the association between ACEs and the use of healthcare. For instance, ACEs are linked with unfavourable socioeconomic circumstances in adulthood, such as lower income or education (Currie \& Widom, 2010), which in turn, are associated with decreased utilisation of healthcare services (Olah et al., 2013). For instance, a study based on a large survey, representative of non-institutionalised adults in the USA, found that ACEs were linked with lower odds of having a physician check-up (Alcala et al., 2018). The hesitancy to access healthcare services may also stem from discomfort associated with feeling of revictimisation (Ackerson, 2012; Cadman et al., 2012) and potential distrust of services based on prior negative experiences (Arias, 2004). Future research could use the 1958 British birth cohort, among other cohorts, to examine potential mechanisms linking ACEs and healthcare utilisation, while accounting for concurrent mental health problems.

If the association between ACEs and mental health outcomes is assumed to be causal, the most effective population health strategy would involve acting early in childhood to prevent ACEs from happening. This would potentially result in saving great economic and social costs due to their link with increased use of healthcare services and medicalisation, among other socioeconomic outcomes. As previously estimated, up to a third of depression and anxiety is attributed to ACEs, with total costs reaching $£ 465.3$ million in Wales and $£ 10.7$ billion in England (Hughes et al., 2020). ACEs are highly prevalent and largely preventable, with a large room for effective interventions, as estimated 2.3 million children in England live in families with complex needs, out of whom only a third receive established support from statutory services (Children's Commissioner for England, 2019). Moreover, ACEs are not only associated with poor mental health but also lower wellbeing. This is an important distinction as wellbeing and mental health outcomes tend to have low or moderate correlations with each other, as found in our study. Hence, the absence of mental illness does not necessarily equate to a happy and fulfilling life and ACEs appear to reduce the ability to live one's life to their full potential.

The main strength of our study is that it used measures of various domains of mental health spanning age 16 to 55, capturing self-reported psychological distress, wellbeing and impact of ACEs on the utilisation of mental health services, however, not all measures under investigation were available at each age. Another limitation was a presence of missing information. To mitigate potential bias due to non-response or attrition, we multiply imputed missing values while including a rich set of predictors of missing information in the imputation model. This allowed for replacing missing data with greater precision (Mostafa \& Wiggins, 2015). Another strength of our study was the inclusion of both prospectively and retrospectively reported measures of ACEs, which tend to identify different groups of individuals (Baldwin et al., 2019). Hence, it is beneficial to examine the sensitivity of findings to both types of measures. However, ACEs captured prospectively and retrospectively had little overlap, with different respondents (e.g., health visitor vs cohort member), preventing direct comparisons. Moreover, the measures of ACEs were often relatively crude, for instance, based on observations by the health visitors who conducted the interviews or self-reported without using standardised and validated tools.

To sum up, we found a strong, graded and persistent link between adverse childhood experiences and a vast array of wellbeing and mental health outcomes, regardless of ACEs being measured prospectively or retrospectively. Our findings reiterate the need to address early-life adverse events to reduce inequalities in a broad range of mental health outcomes across adulthood.

\section{Author contributions}

Dawid Gondek had full access to all of the data in the study and takes responsibility for the integrity of the data and the accuracy of the data analysis.

Study concept and design: Dawid Gondek, Praveetha Patalay, Rebecca Lacey.

Acquisition, analysis, or interpretation of data: Dawid Gondek, Praveetha Patalay, Rebecca Lacey.

Drafting of the manuscript: Dawid Gondek.

Critical revision of the manuscript for important intellectual content: Dawid Gondek, Praveetha Patalay, Rebecca Lacey.

Statistical analysis: Dawid Gondek.

Administrative, technical, or material support: Dawid Gondek, Praveetha Patalay, Rebecca Lacey.

Study supervision: Dawid Gondek, Praveetha Patalay, Rebecca Lacey.

\section{Conflict of interest disclosures}

None reported.

\section{Funding/support}

This study was supported by grant ES/P010229/1 from the Economic and Social Research Council (ESRC).

\section{Declaration of competing interest}

The authors declare the following financial interests/personal relationships which may be considered as potential competing interests: Rebecca Lacey reports financial support was provided by Economic and Social Research Council.

\section{Appendix A. Supplementary data}

Supplementary data to this article can be found online at https:// doi.org/10.1016/j.ssmmh.2021.100013.

\section{References}

Ackerson, K. (2012). A history of interpersonal trauma and the gynecological exam. Qualitative Health Research, 22, 679-688.

Alcala, H. E., Valdez-Dadia, A., \& von Ehrenstein, O. S. (2018). Adverse childhood experiences and access and utilization of health care. Journal of Public Health, 40, 684-692.

Allen, J., Balfour, R., Bell, R., \& Marmot, M. (2014). Social determinants of mental health. International Review of Psychiatry, 26, 392-407.

Arias, I. (2004). Report from the CDC. The legacy of child maltreatment: Long-term health consequences for women. Journal of Women's Health, 13, 468-473.

Arseneault, L., Bryan, B., \& Maughan, B. (2020). Catalogue of mental health measures.

Baldwin, J. R., Reuben, A., Newbury, J. B., \& Danese, A. (2019). Agreement between prospective and retrospective measures of childhood maltreatment. JAMA Psychiatry, 76(6), 584-593. https://doi.org/10.1001/jamapsychiatry.2019.0097

Cadman, L., Waller, J., Ashdown-Barr, L., \& Szarewski, A. (2012). Barriers to cervical screening in women who have experienced sexual abuse: An exploratory study. Journal of Family Planning and Reproductive Health Care, 38, 214-220.

Chang-Quan, H., Xue-Mei, Z., Bi-Rong, D., Zhen-Chan, L., Ji-Rong, Y., \& Qing-Xiu, L. (2010). Health status and risk for depression among the elderly: A meta-analysis of published literature. Age and Ageing, 39, 23-30.

Children's Commissioner for England. (2019). Childhood vulnerability in England 2019. London, UK: Children's Commissioner.

Currie, J., \& Widom, C. S. (2010). Long-term consequences of child abuse and neglect on adult economic well-being. Child Maltreatment, 15, 111-120.

Ege, M. A., Messias, E., Thapa, P. B., \& Krain, L. P. (2015). Adverse childhood experiences and geriatric depression: Results from the 2010 BRFSS. American Journal of Geriatric Psychiatry, 23, 110-114. 
England, C.s. C.f. (2019). Children's commissioner for England. London, UK: Children's Commissioner.

Hughes, K., Bellis, M. A., Hardcastle, K. A., Sethi, D., Butchart, A., Mikton, C., Jones, L., \& Dunne, M. P. (2017). The effect of multiple adverse childhood experiences on health: A systematic review and meta-analysis. Lancet Public Health, 2, e356-e366.

Hughes, K., Ford, K., Kadel, R., Sharp, C. A., \& Bellis, M. A. (2020). Health and financial burden of adverse childhood experiences in England and Wales: A combined primary data study of five surveys. Bmj Open, 10.

Hughes, K., Lowey, H., Quigg, Z., \& Bellis, M. A. (2016). Relationships between adverse childhood experiences and adult mental well-being: Results from an English national household survey. BMC Public Health, 16, 222.

Kalmakis, K. A., \& Chandler, G. E. (2015). Health consequences of adverse childhood experiences: A systematic review. J Am Assoc Nurse Pract, 27, 457-465.

Kelly-Irving, M., Lepage, B., Dedieu, D., Lacey, R., Cable, N., Bartley, M., Blane, D., Grosclaude, P., Lang, T., \& Delpierre, C. (2013). Childhood adversity as a risk for cancer: Findings from the 1958 British birth cohort study. BMC Public Health, 13, 767.

Kuh, D., \& the New Dynamics of Ageing Preparatory Network. (2007). A life course approach to healthy aging, frailty, and capability. Journal of Gerontology: Series A, 62, 717-721.

Lacey, R. E., Pinto Pereira, S. M., Li, L., \& Danese, A. (2020). Adverse childhood experiences and adult inflammation: Single adversity, cumulative risk and latent class approaches. Brain, Behavior, and Immunity, 87, 820-830.

McLaughlin, K. A., Weissman, D., \& Bitran, D. (2019). Childhood adversity and neural development: A systematic review. Annu Rev Dev Psychol, 1, 277-312.

Miller, A. B., Sheridan, M. A., Hanson, J. L., McLaughlin, K. A., Bates, J. E., Lansford, J. E., Pettit, G. S., \& Dodge, K. A. (2018). Dimensions of deprivation and threat, psychopathology, and potential mediators: A multi-year longitudinal analysis. Journal of Abnormal Psychology, 127, 160-170.
Mostafa, T., Narayanan, M., Pongiglione, B., Dodgeon, B., Goodman, A., Silverwood, R., \& Ploubidis, G. (2021). Missing at random assumption made more plausible: Evidence from the 1958 British birth cohort. Journal of Clinical Epidemiology, 136, 44-54. https://doi.org/10.1016/j.jclinepi.2021.02.019.

Mostafa, T., \& Wiggins, R. D. (2015). The impact of attrition and non-response in birth cohort studies: A need to incorporate missingness strategies. Longitudinal and Life Course Studies, 6, 131-146.

Olah, M. E., Gaisano, G., \& Hwang, S. W. (2013). The effect of socioeconomic status on access to primary care: An audit study. Canadian Medical Association Journal, 185, E263-E269.

Peng, P., \& Kievit, R. A. (2020). The development of academic achievement and cognitive abilities: A bidirectional perspective. Child Development Perspectives, 14, 15-20.

Power, C., \& Elliott, J. (2006). Cohort profile: 1958 British birth cohort (national child development study). International Journal of Epidemiology, 35, 34-41.

Selous, C., Kelly-Irving, M., Maughan, B., Eyre, O., Rice, F., \& Collishaw, S. (2020). Adverse childhood experiences and adult mood problems: Evidence from a fivedecade prospective birth cohort. Psychological Medicine, 50, 2444-2451.

Slade, M. (2010). Mental illness and well-being: The central importance of positive psychology and recovery approaches. BMC Health Services Research, 10.

StataCorp. (2020). Stata statistical software: Release 16. College Station, TX: StataCorp LLC.

VanderWeele, T. J. (2019). Principles of confounder selection. European Journal of Epidemiology, 34, 211-219.

VanderWeele, T. J., Mathur, M. B., \& Chen, Y. (2020). Outcome-wide longitudinal designs for causal inference: A New template for empirical studies. Statistical Science, 35, 437-466.

White, I. R., Royston, P., \& Wood, A. M. (2011). Multiple imputation using chained equations: Issues and guidance for practice. Statistics in Medicine, 30, 377-399. 